\title{
ISOCAM data processing
}

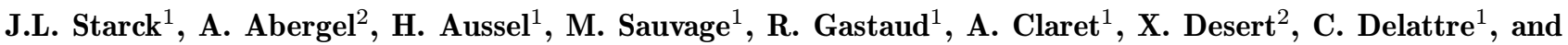 \\ E. Pantin ${ }^{1}$ \\ 1 DAPNIA/SEI-SAP, CEA-Saclay, 91191 Gif-sur-Yvette Cedex, France \\ 2 IAS, Université Paris-Sud, Bât. 121, 91405 Orsay Cedex, France
}

Received November 12, 1997; accepted July 8, 1998

\begin{abstract}
The ISOCAM infrared camera is one of the four instruments on board the ISO (Infrared Space Observatory) spacecraft which was launched successfully on 1995 November 17. It operates in the $2.5-18 \mu \mathrm{m}$ range, and was developed by the ISOCAM consortium lead by the French Service d'Astrophysique of CEA Saclay. This paper presents different algorithms which can be used for the calibration of ISOCAM data. Advantages and limitations of each method are also outlined. A range of examples illustrates the results.
\end{abstract}

Key words: methods: data analysis - instrumentation: interferometers - infrared: general

\section{Introduction}

ISOCAM was designed to provide images of the sky and polarization measurements in the $2.5-18 \mu \mathrm{m}$ band. It features two detectors, one for short wavelengths (SW: $2.5-5.5 \mu \mathrm{m}$ band), the other for long wavelengths (LW: $4-18 \mu \mathrm{m}$ band). The camera has two channels which cannot be used simultaneously: a selection wheel holding Fabry mirrors can direct the light beam from the ISO telescope toward either one of the detectors. The selection wheel also holds two internal calibration sources which can illuminate the detectors quasi-uniformly for flatfield purposes. In order to choose the observing configuration, there are two wheels for each channel. The first wheel holds four lenses allowing the choice of the spatial sampling: $1.5,3,6$, and $12^{\prime \prime}$ per pixel. At ISOCAM wavelengths, the spatial resolution is diffraction limited. The second wheel holds a dozen discrete band pass filters with spectral resolution ranging from 2 to 15 , and continuously variable filters (CVF) with spectral resolution of 45 . The sixth wheel, the entrance wheel, has four positions: one hole and three polarizers. It is possible to observe data

Send offprint requests to: jstarck@cea.fr with different exposure times $(0.28,2.1,5.04,6.02,10.08$, 20.16 and $60.2 \mathrm{~s}$ ) due to the telemetry flow and on-board electronics. The electronic gain can be adjusted to 1,2 or 4. The operating temperature of the camera is as low as $2.4 \mathrm{~K}$, provided by liquid helium cooling. All details about ISOCAM, including in flight performances, are available in Cesarsky et al. (1996).

For one observation, the ISOCAM instrument delivers a set of $32 \times 32$ frame pairs (start of integration, called Reset, and end of integration, EOI). For the long wavelength detector (LW), the signal corresponds to a simple difference between EOI and Reset. For the short wavelength detector (SW), it is more complex, and several operations such as "cross talk correction" must be done. We assume that these corrections have been applied to the data being considered here. We have a set of data noted $D(x, y, t, c)$ : one measurement per pixel position $(x, y)$, repeated $t$ times, with $c$ configurations (there is a new configuration each time the pointing position, the filter, the integration time, etc, are changed).

In the ideal case, calibration will consist of

- normalizing the data to ADU (analog-to-digital units), $\mathrm{g}^{-1} \mathrm{~s}^{-1}$ by

$D_{1}(x, y, t, c)=\frac{D_{0}(x, y, t, c)}{\text { gain } * \text { tint } * \text { Naccu }}$

where gain is the electronic gain, tint is the integration time, $N a c c u$ is the number of frames already added by the on-board processing ( $\mathrm{Naccu}$ is greater than one only in the accumulation mode, normally confined to the 0.28 second readouts $(N a c c u=4)$, or in the CAM parallel mode $(\mathrm{Naccu}=12))$.

- subtract the DARK current

$D_{2}(x, y, t, c)=D_{1}(x, y, t, c)-\operatorname{dark}(x, y)$.

The corresponding dark is extracted from the calibration library.

- divide by the optical flat (oflat) and the detector flat (dflat)

$D_{3}(x, y, t, c)=\frac{D_{2}(x, y, t, c)}{\operatorname{oflat}(x, y) \operatorname{dflat}(x, y)}$. 
The corresponding optical and detector flats are extracted from the calibration library.

- average the values corresponding to the same sky position and the same configuration

$$
\begin{aligned}
& \operatorname{Image}(x, y, c)=\operatorname{mean}\left(D_{3}(x, y, 1 . . t, c)\right) \\
& \operatorname{RMS}(x, y, c)=\operatorname{sigma}\left(D_{3}(x, y, 1 . . t, c)\right)
\end{aligned}
$$

- in the case of raster observations, reconstruct the final raster map, $\operatorname{Raster}(x, y)$, from all images, Image $(x, y, c)$.

In practice, however, we have to take into account several problems:

- since the dark exhibits variation from one orbit to another, library darks obtained on specific calibration orbits are not always applicable to the data, especially at low flux.

- calibration flat fields are made with an internal source, which creates flats different from those of astronomical sources.

- cosmic rays hit the detector (see next section).

- the detector exhibits transient behavior. Each time a detector pixel is illuminated successively by a source and the background, as the detector is scanning the sky, the transition between the two flux levels is not instantaneous.

- Spacecraft jitter around the nominal pointing position randomly shifts the sources on the array during the observation.

- field of view is subject to distortion. The resolution of each pixel depends on its position in the detector.

- no signal is read from Col. 24.

Studies have been done and continue in order to resolve all of these problems, and some solutions have been proposed.

In this paper we present a selection of methods which are commonly used prior to any scientific analysis of the data. Except for the automatic flat field calculation which requires a raster mode, they can be applied to beamswitching, cvf, raster, or polarization measurements. All these methods have been naturally selected by the CAM Instrument Dedicated Team and CAM Instrument Support Team during the last two years of intensive experimentation and data reduction. Other methods also exist, but were found less efficient, or less reliable than those presented here. They are however described in the different CEA and IPAC technical reports given in the reference list.

In the calibration process proposed here, pixels are considered to be independent. This assumption is not true, due to electronic and photonic effects, and the calibration process should be considered as a first approximation. The main area in which this assumption clearly leads to incorrect results is transient correction and, to a lesser extent, cosmic ray removal.

\section{Cosmic ray impact suppression}

\subsection{Cosmic ray impacts}

At first sight, the images from ISOCAM are crossed by strings of high value pixels produced by cosmic ray impacts. This is most apparent for the LW channel of ISOCAM as the LW detector is significantly thicker than the SW detector. Most of these glitches are due to mild, fast electron energy deposition along a string of pixels. Typically for the LW detector, on average, about 40 to 60 pixels are affected at any time for an integration time of 5 seconds. Those pixels usually recover completely after one or two readouts. However, some impacts can have long lasting effects (up to 5 minutes) on the hit pixel. They are thought to be due to heavy particles. There is one impact with this behavior about every second somewhere on the LW channel. The rate of cosmic ray impacts increases greatly when ISO is close to its perigee, due to the radiation belt.

Cosmic ray impact suppression (also called deglitching) is not a trivial task for several reasons. First of all the data are rarely fully stabilized (i.e. it takes a long time until the pixel reaches a stabilized value, although the incoming flux is constant) and this implies that not all differences between two successive frames can be attributed to cosmic ray impacts. Secondly, several glitches can hit the same pixel successively and create a long temporal structure which could be considered a source by a simple algorithm. The wavelet transform is not well adapted to treat this kind of data, due to the linearity of the transform. At a glitch position, a structure would be detected at all scales. This is due to the high intensity of the glitch. The Multiresolution Median Transform (MMT), proposed by Starck et al. (1996), is an alternative to the wavelet transform. It is a non-linear multiresolution transform, and is particularly useful every time we have structures with large dynamics. This is the case for the deglitching problem.

\subsection{The multiresolution median transform}

The median transform is nonlinear, and offers advantages for robust smoothing (i.e. the effects of outlier pixel values are mitigated). We define the median transform of a one dimensional signal $S$, with a window of dimension $n$, as $\operatorname{med}(S, n)$. Let $n=2 l+1$; initially $l=1$. The iteration counter is denoted by $j$, and $N_{\mathrm{s}}$ is the user-specified number of resolution scales.

1. Let $j=1, l=1$, and $c_{1}=S$.

2. Determine $c_{j+1}=\operatorname{med}(S, 2 l+1)$.

3. The multiresolution coefficients $w_{j}$ are defined as: $w_{j}=c_{j}-c_{j+1}$.

4. Let $j \longleftarrow j+1 ; l \longleftarrow 2 l$. Return to Step 2 if $j<N_{\mathrm{s}}$. 
A straightforward expansion formula for the original signal is given by:

$S=c_{\mathrm{p}}+\sum_{j} w_{j}$

where $c_{\mathrm{p}}$ is the residual signal.

In Step 3, the set of resolution levels associated with $l$ leads to a dyadic analysis. Other possibilities involving intermediate scales (e.g. $l \longleftarrow 1.5 l$ ) can also be considered.

The multiresolution coefficient values, $w_{j}$, are not necessarily of zero mean. Note that values of $w_{j}$ can be negative.

For integer signal input values, this transform can be carried out in integer arithmetic alone which may lead to computational savings.

Computational requirements of the multiresolution transform are high, and these can be reduced by decimation: one pixel out of two is retained at each scale. Here the transform kernel does not change from one iteration to the next, but the signal to which this transform is applied does. This pyramidal algorithm is described in Starck et al. (1998).

\subsection{Cosmic ray impact suppression}

As the glitch structures can have different sizes, we need a multiresolution tool in order to perform efficient automatic detection. The idea developed here is the following: as we observe the same position in the sky during $n$ exposures, we cannot have any structure in our signal which has a temporal size less than $n *$ tint (tint being the exposure time of a single frame). It means that all the significant structures (i.e. not due to the noise) at small temporal scales are due to the glitches.

The method consists in doing for each pixel $(x, y)$ the following steps, where $D$ represents the raw data (three dimensional array)

1. set $S(t)=D(x, y, t)$

2. estimate the temporal noise $\sigma_{\mathrm{t}}$ in $S(t)$ (see (Starck \& Murtagh 1998) for more details on this step)

3. calculate the MMT of $S(t)$. The result is $w_{j}(t)$ where $j$ is the temporal scale. The number of scales in the transform is fixed due to the number of frames per satellite position.

4. derive from $\sigma_{\mathrm{t}}$ the standard deviation $\sigma_{j}$ at each scale $j$.

5. at all scales, set to zero all structures higher than a given level.

$$
\text { if } \operatorname{abs}\left(w_{j}(t)\right)>k \sigma_{j} \text { then } w_{j}(t)=0
$$

$k$ is generally taken equal to 4 .

Figure 1 shows the results of such a treatment. Figure 1 (top) shows the values of a pixel of the camera as time elapses. The $x$-axis represents the frame number (time/integration time), and the $y$-axis is the signal in
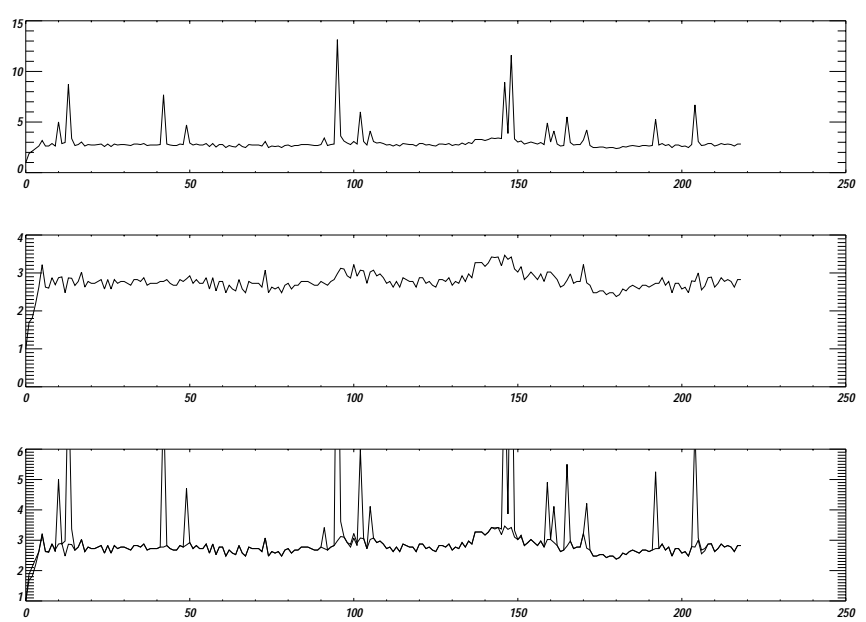

Fig. 1. Original data (top), deglitched data (middle), and both overplotted (bottom)

ADU per second. These data were collected during a raster observation, and the satellite remained at the same position for about 20 frames, and the integration time was equal to $2.1 \mathrm{~s}$. A source is at the limit of detection (frames 130 to 150). All peaks are due to cosmic ray impacts. The intensity of a cosmic ray can range all values between a few ADU to more than one thousand ADU (glitch detection limit depends on the noise level in the data). Figure 1 (middle) shows the same data after the glitch suppression. The third plot (Fig. 1 (bottom)) shows both data and deglitched data overplotted. We see that the noise and the signal are not modified during this operation.

The method is robust and works for non-stabilized data. The only real limitation is that we cannot detect glitches which last for a time longer than or equal to $n *$ tint. That means that the more frames we have per camera configuration, the better the deglitching will be. Some "special" glitches introduce a gain variation with a very long time duration. These special glitches can be separated in two types: 1) the pixel value decreases slowly until a stabilized value is reached (see Fig. 2); 2) the pixel value decreases first below the stabilized value, and then increase slowly until the stabilized value is reached (see Fig. 3). In both cases, the stabilization can be very slow, and the deglitching method presented here does not correct for this effect. Finally, pixels where a glitch has been detected are not used when averaging values corresponding to the same sky position and same configuration.

The deglitching works separately on each pixel, and does not take into account the spatial information. In principle it should identically deglitch point sources and extended sources. However, in practice, differences are introduced by the spacecraft jitter, especially when using the $1.5^{\prime \prime}$ and $3^{\prime \prime}$ lenses. As jitter will slightly move point sources on the array, which exhibit strong brightness spatial gradients, this random motion creates artificial 


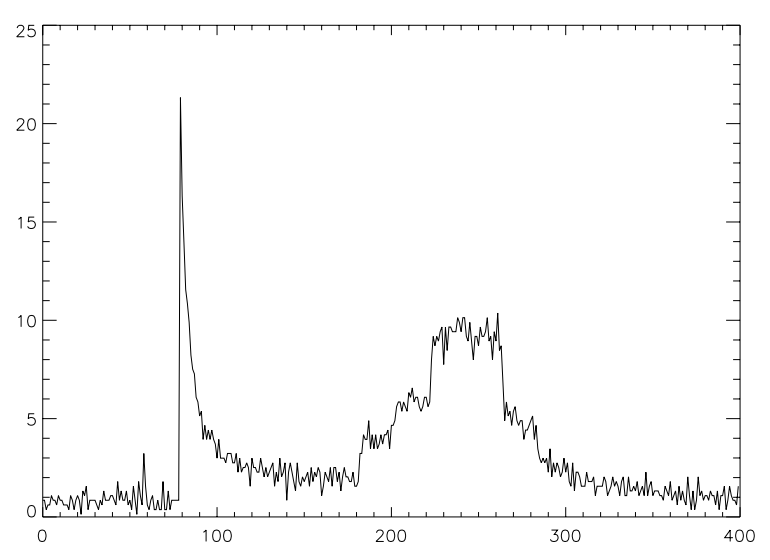

Fig. 2. Glitch with very long duration. The flux in ADU is plotted against time given by the exposure index

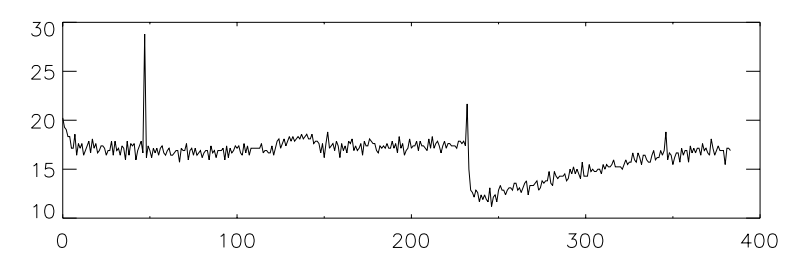

Fig. 3. Glitch with a negative tail. The flux in ADU is plotted against time given by the exposure index. Note the gain variation of about 5 ADUs which appears after the second glitch

temporal variations similar to glitches on any pixel that sees the sources, although in these cases they correspond to real signal. Thus these variations are generally removed by the deglitching algorithms and the consequence is a systematic loss of flux for point sources. No automatic procedure currently exists to account for this artifact.

\section{Dark subtraction for the LW channel}

One has to subtract the dark current from the image, for both the SW and LW channels. This is done with measurements obtained during dedicated calibration orbits. This procedure produces acceptable results for the SW channel, but can sometimes fail for the LW channel. The reason for this is a combination of long-term drifts in the dark current, and low-signal that will make these drifts dominate the noise over photon and readout noise. This situation is quite easy to recognize as the LW dark current shows strong odd-even stripes (see Fig. 4), which are not completely gone when the dark correction fails. To provide the reader with orders of magnitudes for these effects, Table 1 lists, for all integration times, the spatial mean of the noise on the calibration dark measurement (each dark is the result of the average of a given number of frames, therefore each pixel in the dark measurement has an associated RMS, we report here the mean of these RMS), and, separately for the even and odd lines, the mean, median and rms of the dark level. Finally, this table also lists typical values of residuals (mean and rms) that can be obtained when the calibration dark is used (see also Table 2). These values were obtained by correcting very long dark measurements by the calibration dark. These long measurements are performed to derive the time behavior of ISOCAM LW dark (Biviano et al. 1998). As can be seen from the table, the dark correction is not perfect and there remains a residual whose amplitude is larger than the noise in the calibration dark. Furthermore a clear even-odd pattern remains (see Fig. 4) as indicated by the relatively large dispersion.

The offset between the calibration dark and the actual dark can only be derived from a study of the time behavior of the dark (Biviano et al. 1998). The pattern, however, can be removed with appropriate analysis (e.g. Van Buren \& Aussel 1996; Starck \& Pantin 1996).

\subsection{Dark pattern removal using the maximum entropy method (MEM) filtering}

In order to extract the residual dark from the data, we first derive the median image $M(x, y)$ by taking the median of all values Image $(x, y, *)$ for a given detector pixel. If the pixel $(x, y)$ sees the background longer than an object, then $M(x, y)$ (renormalized) gives a good estimate of the flat-field at this position. In a general way, $M(x, y)$ contains less signal than $I(x, y, c)$ for any configuration $c$, and we prefer to try to extract the residual dark in $M$ than in $I$.

Filtering can be applied to $M$ to suppress the visual residual dark. In order to achieve this without modifying the signal significantly, we use the vertical cross-entropy of image $O(x, y)$ defined by

$$
\begin{aligned}
E(O)= & \sum_{x, y}(O(x, y)-O(x, y-1))+(O(x, y)-O(x, y+1)) \\
& -O(x, y) \ln \left(\frac{O(x, y)^{2}}{|O(x, y-1) O(x, y+1)|)}\right) \cdot
\end{aligned}
$$

This entropy definition leads to a solution where the difference between pixels in one direction is minimized while matching the data as closely as possible.

The functional to minimize is:

$J(F)=\frac{\|M-F\|^{2}}{2 \sigma^{2}}-\alpha E(F)$

in which the first term $\left(\frac{\|M-F\|^{2}}{2 \sigma^{2}}\right)$ represents the "goodness of fit" (GOF) constraint, which is regularized by the vertical cross-entropy functional $E(F) . \sigma$ is the noise standard deviation, and $\alpha$ a parameter defining the weight between the GOF term and the regularizing efficiency by the cross-entropy. $F$ is the filtered image. 
Table 1. Dark levels and associated dispersions for the LW channel of ISOCAM. Column (1) is the integration time in seconds, $(2)$ is the spatial mean of the noise $(1 \sigma)$ in the calibration dark measurement. As the LW dark shows a strong separation between the odd and even lines (due to the different amplification chains), we have listed the levels separately for the even and odd lines. The mean dark value, the median dark value, and the $1 \sigma$ dispersion around the mean are listed for the even lines (Cols. 3 to 5) and odd lines (Cols. 6 to 8). Finally in the last two columns, we have used data obtained during "dark" revolutions (CAM is kept closed for a whole revolution and is continuously read out) to exemplify the amplitude of dark drifts by subtracting the calibration dark from these dark measurements. The spatial mean and rms are listed in Cols. (9) and (10). All dark values are in $\mathrm{ADU} \mathrm{g}^{-1} \mathrm{~s}^{-1}$. No values are listed for the $0.28 \mathrm{~s}$ integration time since due to technical constraints, it cannot be measured for a complete orbit

\begin{tabular}{|c|c|c|c|c|c|c|c|c|c|}
\hline \multirow{2}{*}{$\begin{array}{r}T_{\mathrm{int}}(\mathrm{s}) \\
(1)\end{array}$} & \multirow{2}{*}{$\begin{array}{c}\sigma_{\mathrm{N}} \\
\underset{(2)}{\operatorname{ADU} g^{-1} \mathrm{~s}^{-1}}\end{array}$} & \multicolumn{3}{|c|}{ Even Lines } & \multicolumn{3}{|c|}{ Odd Lines } & \multicolumn{2}{|l|}{ Dark n } \\
\hline & & $\begin{array}{r}\text { Mean } \\
\text { (3) }\end{array}$ & $\begin{array}{r}\text { Median } \\
\text { ADU g }^{-} \\
(4)\end{array}$ & $\mathrm{rms}$ & $\begin{array}{r}\text { Mean } \\
\quad(6)\end{array}$ & $\begin{array}{c}\text { Median } \\
\text { ADU g }^{-1} \\
(7)\end{array}$ & $\mathrm{rms}$ & $\begin{array}{r}\text { Mean } \\
\text { ADU } \\
(9)\end{array}$ & $\begin{array}{l}\mathrm{rms}^{-1} \\
(10)\end{array}$ \\
\hline 0.28 & 0.66 & -46.7 & -43.5 & 10.9 & -28.4 & -26.4 & 6.0 & & \\
\hline 2.1 & 0.16 & -10.3 & -9.87 & 1.53 & -7.08 & -6.83 & $8.1510^{-1}$ & -0.58 & 0.27 \\
\hline 5.04 & $7.010^{-2}$ & -4.87 & -4.68 & $6.9510^{-1}$ & -3.63 & -3.53 & $3.5510^{-1}$ & -0.31 & 0.16 \\
\hline 10.08 & $4.610^{-2}$ & -2.57 & -2.48 & $3.6310^{-1}$ & -2.04 & -1.98 & $1.8810^{-1}$ & -0.18 & 0.07 \\
\hline 20.16 & $3.610^{-2}$ & -1.25 & -1.21 & $1.9210^{-1}$ & -1.04 & -1.02 & $1.0510^{-1}$ & -0.16 & 0.05 \\
\hline
\end{tabular}
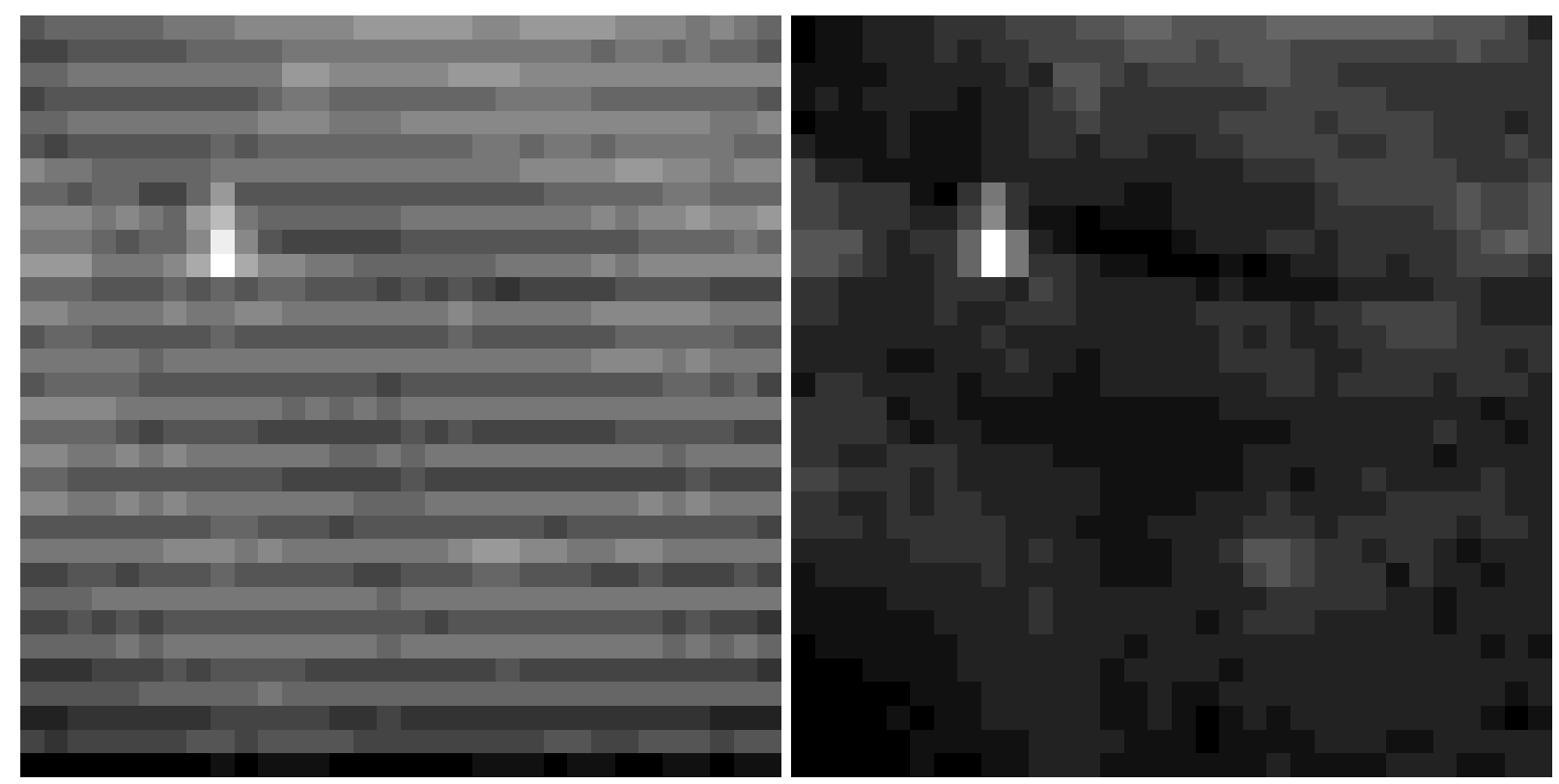

Fig. 4. Image before dark subtraction (left) and after (right). Notice the dark pattern, which is visible as a change of the signal by comparing odd and even lines in the original image

The gradient of the former functional is

$$
\begin{gathered}
\nabla(J(F(x, y)))=-\frac{(M-F)(x, y)}{\sigma^{2}} \\
+\alpha \operatorname{sgn}(F(x, y)) \ln \left(\frac{F(x, y)^{2}}{|F(x, y-1) F(x, y+1)|}\right) .
\end{gathered}
$$

Then the "one step gradient" algorithm gives us an iterative scheme to minimize the functional (4):

$F^{n+1}=F^{n}-\gamma \nabla\left(J\left(F^{n}\right)\right)$.

The residual dark is finally obtained by taking the difference between $M$ and $F$.

\subsection{Dark pattern removal in Fourier space}

The dark pattern can be suppressed in Fourier space by the following method:
1. Average together all deglitched frames, obtaining $I_{\mathrm{a}}$.

2. Eliminate in $I_{\mathrm{a}}$ the low frequencies, obtaining $I_{\mathrm{h}}$.

3. Estimate the noise in $I_{\mathrm{h}}$, and set to zero all structures higher than three times the noise standard deviation.

4. Compute the FFT $\hat{I}_{\mathrm{h}}$ of $I_{\mathrm{h}}$, and estimate the noise in the real part $\hat{I_{\mathrm{h}}}$, and imaginary part $\hat{I}_{\mathrm{h}}$ of $\hat{I}_{\mathrm{h}}$.

5. Threshold all Fourier coefficients lower than the noise. We get $\hat{T}_{\mathrm{hr}}, \hat{T}_{\mathrm{hi}}$.

6. Compute the inverse FFT transform of $\left(\hat{T}_{\mathrm{h}_{\mathrm{r}}}, \hat{T}_{\mathrm{h}_{\mathrm{i}}}\right)$. Its real part gives the pattern $P$. The pattern $P$ can then be subtracted from the input image.

This procedure can be iterated and usually three cycles is sufficient for a good dark pattern removal. 
The residual dark can be relatively well suppressed just by deleting some frequencies. The result is obviously not as good as if we had had the true dark, and there will be always a confidence interval on the flux. Yet the advantage of the FFT thresholding method is that it always finds a residual dark image evaluation with zero mean (whithin the numerical errors). Therefore, the method just suppresses the visual artifacts, without adding any offset to the data. The MEM method produces good results as well, but seems to have some limitations. For instance, it is a real filtering method (even if it is only in one direction), thus, the noise statistics can be modified. This point could be resolved by previous filtering of the data cube. Note, also, that some columns can show atypical behavior and the resulting artifacts seem to be satisfactorily removed when using the FFT thresholding method.

Figure 5 shows the final calibrated raster image of the Antennae, without any second order dark correction (upper), and with a second order dark correction using the FFT method (lower). The visual aspect of the residual dark has disappeared. It must be clear that the "real" dark is not corrected using this method, only its visual aspect is removed.

To provide quantitative information on the quality of this correction we performed the following experiment: we used dark measurements performed during "dark orbits" (see Biviano et al. 1998) and subtracted the corresponding calibration dark from these measurement. The FFT method was applied to the residuals. In Table 2 we give, for the most often used integration times of 2.1, 5.04 and $10.08 \mathrm{~s}$, the mean and rms around the mean for the residuals after calibration dark removal, the mean and rms around the mean for the residuals after the FFT dark correction. As can be seen from the table, the mean value of the residuals is almost unchanged after the FFT dark correction while the rms has been divided by two. More striking is the effect in even-odd pattern. In the last two columns of Table 2 we list the difference between the mean of the even lines and the mean of the odd lines for the residuals after calibration dark correction and those after application of the FFT dark correction. After FFT dark correction the remaining difference becomes barely significant.

\section{Flat field correction}

The flat field is separated in two in the calibration library: the optical flat and the detector flat. The goal of this separation is to minimize the number of configurations to calibrate. The data must be divided by the two flats. However, the library flat fields rarely correspond exactly to the observer's flat field as there exists a small jitter in the position of the wheels of the instrument. This jitter is intentional and was introduced to prevent the wheels from getting stuck. This means that for two observations with the same configuration, the optical flat field can be significantly different. Different methods have been tested in order to derive a flat from the data, but they apply only in the case of a raster observation.

In some cases, particularly for observations using a raster mode on faint sources embedded in a flat extended emission, e.g the zodiacal light, a flat field can be derived from the data by choosing interactively a region where there is no object, and only background. For an automatic flat field estimation, we take advantage of the fact that, in such observations, all pixels of the detector spend more time observing the background than the object, and we apply the following procedure:

1. Average all frames belonging to the same configuration. This gives one image per configuration, i.e. a cube of averaged data $C_{\mathrm{a}}(x, y, c)$.

2. For each pixel $(x, y)$ of the detector, take the median of $C_{\mathrm{a}}(x, y, *)$. This results in $M(x, y)$.

3. The flat is equal to $M$ normalized by the mean of its center: flat $=M / \operatorname{mean}(M(10: 21,10: 21))$. This is the normalization used in all automatic and calibration data reduction, it therefore has to be employed in order to keep the data compatible with, for example, the ADU to mJy conversion factor.

The automatic flat field method generally produces good results. Its only limitation is the assumption that all pixels of the detector spend more time observing the background than the object. It will certainly not always be true for all observations, even in raster mode, and then the procedure will fail.

For raster observation, the flat field estimation can be improved by an iterative method, using the fact that a sky point is seen by different pixels (Starck et al. 1996; van Buren 1996).

\section{Iterative flat field estimation for raster with overlap}

\subsection{Introduction}

In order to improve the estimated flat field, we can use the fact that a sky point is seen by several pixels of the detector. We consider:

$-R=$ number of raster positions

$-a=32^{2}$ number of pixels of the detector

$-u=$ detector overlap ( $u=0$ for no pixel overlap, and $u=1$ for pixel overlap)

$-N=$ total number of pixels in the final image.

The number of unknown variables $X$ should be less than the number $D$ of data. We have $X=N+a$ (final image + flat), and the number of data is equal to $D=R \cdot a$. The final image size depends on both the overlap factor and the number of raster positions:

$N=a+a(R-1)(1-u)=a(R-u R+u)$ 
Table 2. Quantitative information on the performances of the FFT dark subtraction method. This table compares the quality of dark current correction between the standard method, i.e. using a library dark, and the standard+FFT method, where the subtraction of a library dark is followed by FFT filtering. To make this comparison, we are using dark measurements (typically between 10 and 20 per integration times) obtained during "dark" orbits (Biviano et al. 1998). Column (1) gives the integration time in seconds. We only display results for the most commonly used integration times. Columns (2) and (3) list the spatial mean and rms on the library dark corrected images. Columns (4) and (5) give the same information once these images have been FFT filtered. One can see that the mean signal is little affected $(<2 \%)$ while the rms is divided by $\sim 2$. In Cols. (6) and (7) we compare the even and odd lines of the images at the two stages of dark correction by computing the difference between the mean of the even lines and the mean of the odd lines. One can see that while this difference is quite significant after only the library dark correction, it is almost insignificant after FFT filtering

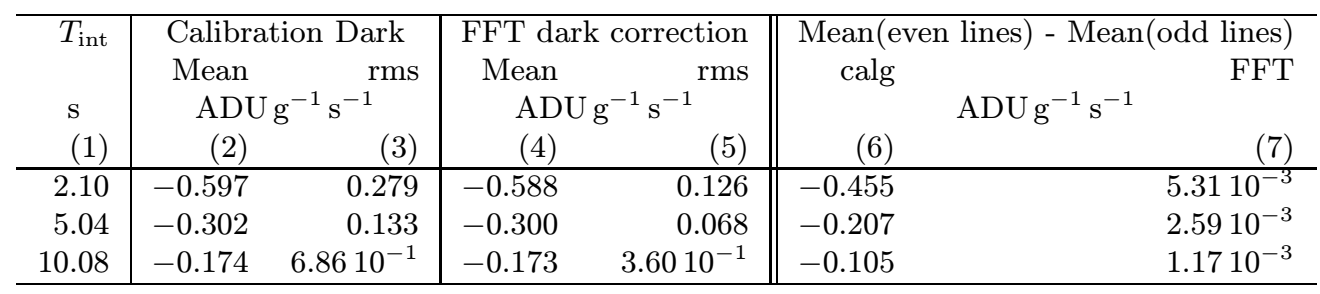
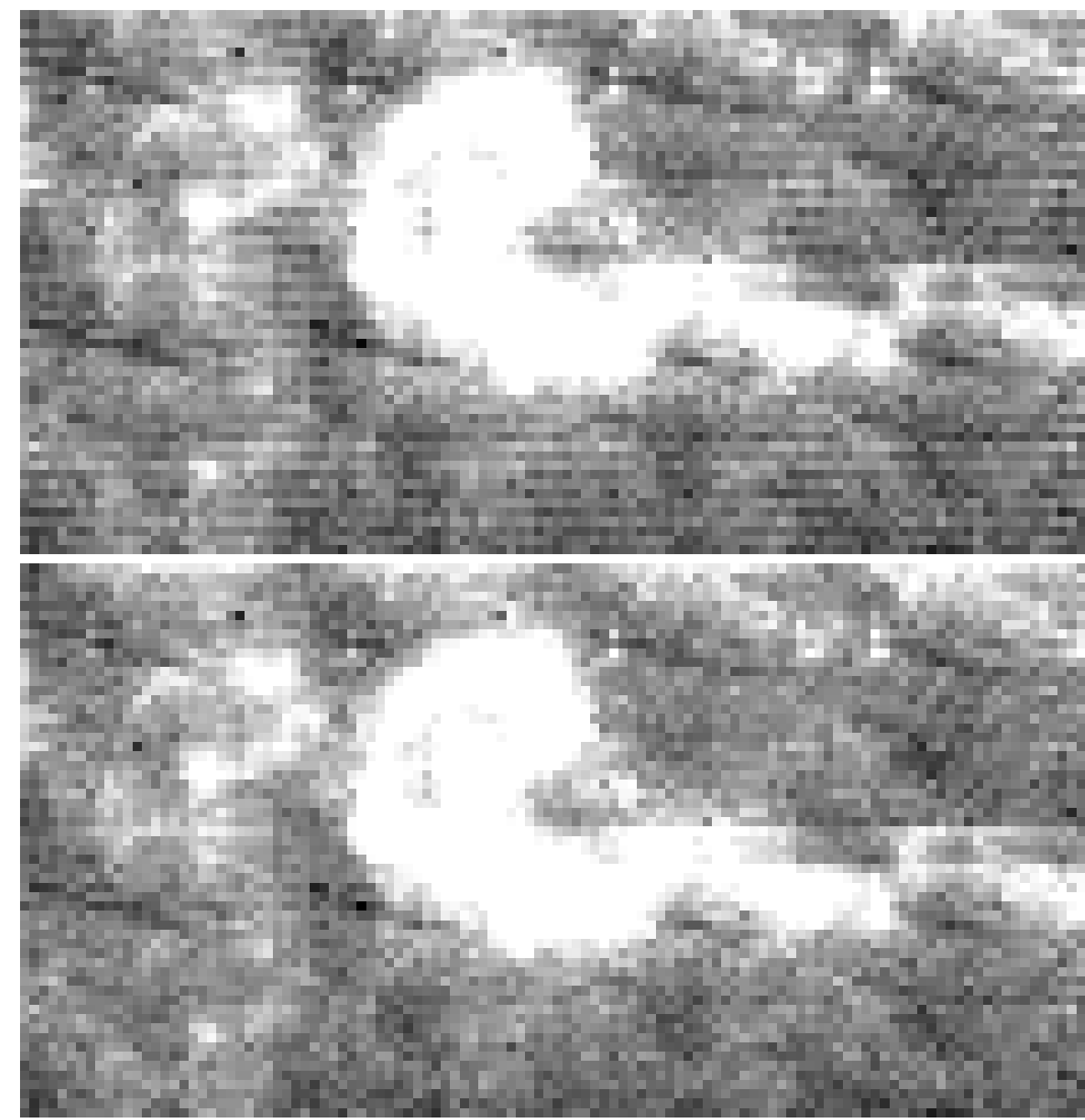

Fig. 5. Upper, raster image of the antennae without second order dark correction, and lower, the same image but using the FFT thresholding second order dark correction 
then $X=a(R-u R+u)+a \leq R a$ implies that

$R \geq \frac{1+u}{u}$

or

$u \geq \frac{1}{R-1}$.

These relationships give only an idea of the limits, because they do not take into account effects such as transients, field of view distortion, noise, etc. As a rule of thumb, one can consider that in practice an increased number of raster positions and pixel overlap will result in a better determination of the system.

\subsection{Iterative flat-field estimation}

We represent the reduced data (without flat field correction) by Image $(x, y, c)$, where $x, y$ is the position on the detector, and $c$ the raster position number or configuration number. From Image and from an initial flat flat ${ }_{0}$, we can build a raster image $R_{0}(k, l)$.

$P\left(\frac{\text { Image }}{\text { flat }_{0}}\right)=R_{0}$.

From the sky image $R_{0}$, we can simulate our data by applying what we call an inverse projection. We obtain the set $M(x, y, c)$

$M(x, y, c)=P^{-1}\left(R_{0}(k, l)\right)$

$M$ can be considered a model of what we should get on the detector if the true sky image is $R_{0}$. This model can be used to obtain an estimation (flat $)_{1}$ ) of the flat. This new flat will then lead to a new raster image, $R_{1}$

$P\left(\frac{\text { Image }}{\text { flat }_{1}}\right)=R_{1}$

or in a more general way, we have the iterative flat field correction

$P\left(\frac{\text { Image }}{\text { flat }_{\mathrm{n}+1}}\right)=R_{n+1}$.

The problem is now to find flat ${ }_{n+1}$ knowing Image and our sky model $M$. The least square solution gives

flat $_{n+1}(x, y)=\frac{\sum_{c} \frac{\operatorname{Image}(x, y, c) M(x, y, c)}{\operatorname{RMS}(x, y, c)^{2}}}{\sum_{c} \frac{M(x, y, c)^{2}}{\operatorname{RMS}(x, y, c)^{2}}}$

Furthermore, we can assume that the flat is varying with the time. Then the Eq. (13) becomes

$P\left(\frac{\text { Image }(x, y, c)}{\operatorname{flat}_{n+1}(x, y, c)}\right)=R_{n+1}$

and if we assume a linear variation constant over the whole image, we have

$P\left(\frac{\text { Image }(x, y, c)}{\alpha_{n+1}(c) \text { flat }_{n+1}(x, y)}\right)=R_{n+1}$ we now have one more unknown. At each iteration, we compute $\alpha_{n+1}$, and flat ${ }_{n+1}$ by

$$
\begin{aligned}
\text { flat }_{n+1}(x, y) & =\frac{\sum_{c} \frac{\alpha_{n}(c) \operatorname{Image}(x, y, c) M(x, y, c)}{\operatorname{RMS}(x, y, c)^{2}}}{\sum_{c} \frac{\alpha_{n}(c) M(x, y, c)^{2}}{\operatorname{RMS}(x, y, c)^{2}}} \\
\alpha_{n+1}(c) & =\frac{\sum_{x, y} M(x, y, c) \mathrm{flat}_{n}(x, y)}{\sum_{x, y} \operatorname{flat}_{n}(x, y)^{2}}
\end{aligned}
$$

at each iteration, the flat and $\alpha$ are normalized

$$
\begin{aligned}
& \text { flat }_{n+1}(x, y)=\frac{\text { flat }_{n+1}(x, y)}{\operatorname{mean}\left(\text { flat }_{n+1}(10: 21,21: 21)\right)} \\
& \alpha_{n+1}(c)=\alpha_{n+1} \text { (Last_Config). }
\end{aligned}
$$

Simulations showed that the flat error can be divided by a factor of two (in our simulations, we derived a flat with a $5 \%$ accuracy, starting with a flat where the error was $10 \%)$. The parameter $\alpha$ is particularly useful for the first configuration when the detector is not stabilized at all.

\section{Stabilization}

\subsection{Introduction}

The response of each CAM-LW pixel strongly depends previous observations. A long-term transient response after changes in photon flux levels is a well-known characteristics of extrinsic IR photoconductors working under low background conditions (see for instance Fouks \& Schubert 1995; Schubert et al. 1995; Haegel et al. 1996 and references therein). The detector used in the LW channel of ISOCAM is a gallium doped silicon photoconductor hybridized by indium bumps. The pixel pitch is $100 \mu \mathrm{m}$ and the detectors are $500 \mu \mathrm{m}$ thick. A physical model has been developed for the Si:Ga detector arrays used in the PHT$\mathrm{S}$ instrument of the ISOPHOT experiment on board ISO (Fouks \& Schubert 1995; Schubert et al. 1995). However, a physical approach to correct ISOCAM data is extremely difficult because of (1) the strong assumptions used to resolve the general equations and (2) the limited number of frames per sky position or CVF spectral position delivered by ISOCAM which generally does not allow any physical model adjustment. As detailed in Abergel et al. (1998), the response of CAM-LW pixels is known to depend on the amplitude of the step, the flux history, the direction of the flux step (upward or downward) and the local spatial gradient of illumination. We present in this section an empirical method, based on a simplified description of the pixel response after a change of the incident flux level. The spatial charge coupling between pixels is neglected.

\subsection{Description of the CAM-LW response}

Ground-based and in-flight measurements have shown that the pixel response after a change of the incident flux level can be separated at least in the following two phases: 
- an instantaneous step to $60 \%$ of the flux step.

- a long variation for the remaining $40 \%$, in a first order exponential similar to a time constant inversely proportional to the incident flux level.

The exponential description of the long variation for the remaining $40 \%$ after the instantaneous step is an approximation. Going from a dark level to a strong incident flux level (typically of the order of or higher than the zodiacal emission observed with the LW10 broad-band filtered centered at $12 \mu \mathrm{m}$, with an integration time of $2.1 \mathrm{~s}$ ), the first readouts after the instantaneous step strongly depart from an exponential curve. This is likely due to charge coupling between pixels, which can also be responsible for the oscillations that can affect the response curve. These effects are dramatic for strong steps of flux, especially at low background. Therefore, all methods based on an exponential-like description of the pixel response fail for all steps going from the dark level, and for strong steps going from a low background. There is also a very long-term transient which affects typically $5-10 \%$ the flux above the dark level which will not be discussed in this paper. This transient can introduce a memory effect with an amplitude of a few $\%$ of the input flux level, thus affecting the data over several hours.

If the number of readouts per sky position or spectral CVF position is large enough to show a significant fraction of the transient curve, it is possible to fit pixel per pixel the temporal response using a specific function to derive as well as possible a "stabilized" value. Several methods have been developed based on the fit, and we found them less reliable when considering the flux, than the inversion method described in the following (see Sect. 6.6). Furthermore, for most of the observations, only the first part of the transient curve is observed because of the low IR emission of the sources observed with ISOCAM (especially using the CVF). The LW detector will thus never reach any stabilized value during the observation. The principle of the method presented here and developed by Abergel et al. (1996) consists in inverting a simple model of the CAM-LW response in order to recover frame by frame the successive stabilized values.

\subsection{Simplified model of the CAM-LW response}

The idea is to describe the response $s(t)$ of one given pixel as a function of the input flux $i(t)$ (in units of $\mathrm{ADU} \mathrm{g}^{-1} \mathrm{~s}^{-1}$, i.e. proportional to the incoming flux density, dark subtracted but not flat-field or distortion corrected):

$s(t)=r i(t)+(1-r) \int_{-\infty}^{t} i\left(t^{\prime}\right) \frac{\mathrm{e}^{-\frac{t-t^{\prime}}{\tau}}}{\tau} \mathrm{d} t^{\prime}$ with the time constant $\tau$ (in seconds) inversely proportional to the input flux:

$\tau=\frac{\alpha}{i\left(t^{\prime}\right)}$.

It is easy to verify that, for a step of flux going from $i_{1}$ to $i_{2}$, this formula gives an instantaneous response equal to to $r \times\left(i_{2}-i_{1}\right)$, followed by an exponential variation for the remaining $(1-r) \times\left(i_{2}-i_{1}\right)$, with two time constants inversely proportional to $i_{1}$ and $i_{2}$.

From preliminary fitting of the model with the transient responses recorded during a dedicated orbit (revolution 16, during the Performance Verification phase, where cycles of filter sequences were performed), the values of $r$ and $\alpha$ have been adjusted to $r=0.6$ and $\alpha=$ $1200 \mathrm{~s}\left(\mathrm{ADU} \mathrm{g}^{-1} \mathrm{~s}^{-1}\right)^{-1}$ (uncertainties are around 10\%). The low signal to noise ratio has not allowed the detection of any significant variations of these values between pixels, though these are likely to occur.

The camera is not read continuously but rather we obtain a discrete series of readouts for all $t_{j}$. Thus we can rewrite Eq. (19) in this way:

$S\left(t_{i}\right)=r I\left(t_{i}\right)+(1-r) \sum_{j=-\infty}^{i-1} \int_{t_{j}}^{t_{j+1}} I\left(t_{j}\right) \frac{\mathrm{e}^{-\left(\frac{t_{i}-t^{\prime}}{\tau_{j}}\right)}}{\tau_{j}} \mathrm{~d} t^{\prime}$

with $\tau_{j}=\alpha / I\left(t_{j}\right)$.

During an integration, it is assumed (1) that one given pixel sees the same point in the sky and (2) that the configuration of ISOCAM is constant. The effects due to the jitter during the integration are neglected. Thus $I(t)$ is constant between $t_{j}$ and $t_{j+1}$ and the integral of Eq. (21) can be computed for all the terms in the summation. We have:

$S\left(t_{i}\right)=r I\left(t_{i}\right)+(1-r) \sum_{j=-\infty}^{i-1} I\left(t_{j}\right) \mathrm{e}^{\frac{t_{j}-t_{i}}{\tau_{j}}}\left(\mathrm{e}^{\frac{t_{j+1}-t_{j}}{\tau_{j}}}-1\right)$

This simplified model allows us to reproduce the response after a step of flux with a precision of typically $5-10 \%$. It fails especially for large flux steps (typically $\left.80 \mathrm{ADU}^{-1} \mathrm{~s}^{-1}\right)$ : upward transients strongly depart from a simple exponential behavior due to unpredicted oscillations, while downward transients seem to be described more precisely by an hyperbolic curve rather than with an exponential curve. The method is in fact not adapted to measure the brightness of strong point sources, since it neglects the spatial charge coupling, and no methods actually exists allowing this kind of transient to be correctly treated.

\subsection{Inversion of the simplified model of the CAM-LW response}

The transient correction consists of computing the successive values of $I\left(t_{j}\right)$ from the successive CAM readouts $S\left(t_{j}\right)$. It is important to note that $r$ and $\alpha$ are fixed parameters of the model: this method does not use any fitting 
and can be applied whatever the state of the camera and the flux step are. The idea is to invert the Eq. (22), which can easily be done with the two following assumptions:

1. We do not know the values of CAM readouts $S\left(t_{j}\right)$ from $t=-\infty$, since the time series is in the general case cut at the beginning of each observation (at $\left.t=t_{0}\right)$. It is thus necessary to assume a realistic history of the input flux before the first readout of the observation to proceed further. For all the illustrating tests presented in this paper (Figs. 6 and 7), we have assumed that a constant input flux has been observed from $t=-\infty$ up to the first readout (at $t=t_{0}$ ), which means that the camera was assumed to be stabilized before the first readout. This translates in starting our summation at $j=0$ and adding to Eq. (22) a constant (derived from Eq. (19)).

2. In Eq. (22), for the different values of $j$, we have $\tau_{j}=\alpha / I\left(t_{j}\right)$. The successive values of $\tau_{j}$ are not known since they depend on the successive $I\left(t_{j}\right)$. Thus, to allow a simple inversion of the equation, we have assumed that $\tau_{j}=\alpha / S\left(t_{j}\right)$. This assumption obviously leads to inappropriate corrections since $S$ and $I$ can be much different due to the transient effect. A detailed assessment of the impact of this approximation on the photometric accuracy is yet to be made. An alternative approach would be to first consider that $\tau_{j}=\alpha / S\left(t_{j}\right)$ and compute an estimate of the successive values of $I\left(t_{j}\right)$, and then to iterate.

If our observation is made of $\mathrm{N}$ readouts obtained at time $t_{0}, t_{1}, \ldots, t_{N-1}$, we can rewrite Eq. (22) for each pixel in a matrix of the form: $S=[M] \times I+C$. $S$ is the vector of $N$ readouts, i.e. the data, $I$ is the vector of $N$ intensities, i.e the data corrected for transients, $[M]$ is an $N \times N$ transfer matrix whose elements $M_{i, j}$ are such that:

- if $j>i$, then $M_{i, j}=0$,

- if $j=i$, then $M_{i, j}=r$,

- if $j<i$, then $M_{i, j}=(1-r) \mathrm{e}^{\frac{t_{j}-t_{i}}{\tau_{j}}}\left(\mathrm{e}^{\frac{t_{j+1}-t_{j}}{\tau_{j}}}-1\right)$,

and $C$ is is a vector of constants due to assumption (1) such that for all $i, C_{i}=(1-r) I\left(t_{0}\right) \mathrm{e}^{\frac{t_{0}-t_{i}}{\tau_{0}}}$.

Thus for all instants $t_{i}$ one has $S\left(t_{i}\right)=\sum_{j=0}^{N-1} M_{i, j} \times$ $I\left(t_{j}\right)+C_{i}$.

The transient correction consists in computing $I=$ $[M]^{-1} \times(S-C)$, which is always possible since the determinant of the matrix $[M]$ is never equal to zero (all elements above the main diagonal are equal to 0 ). If the correction is perfect, the vector $I$ represents the successive values measured by a detector with no memory effect.

This method of correction allows us to derive in a systematic way, a first order estimate of the sky brightness observed with ISOCAM-LW, without any parameter adjustment. However, one should say right away that the
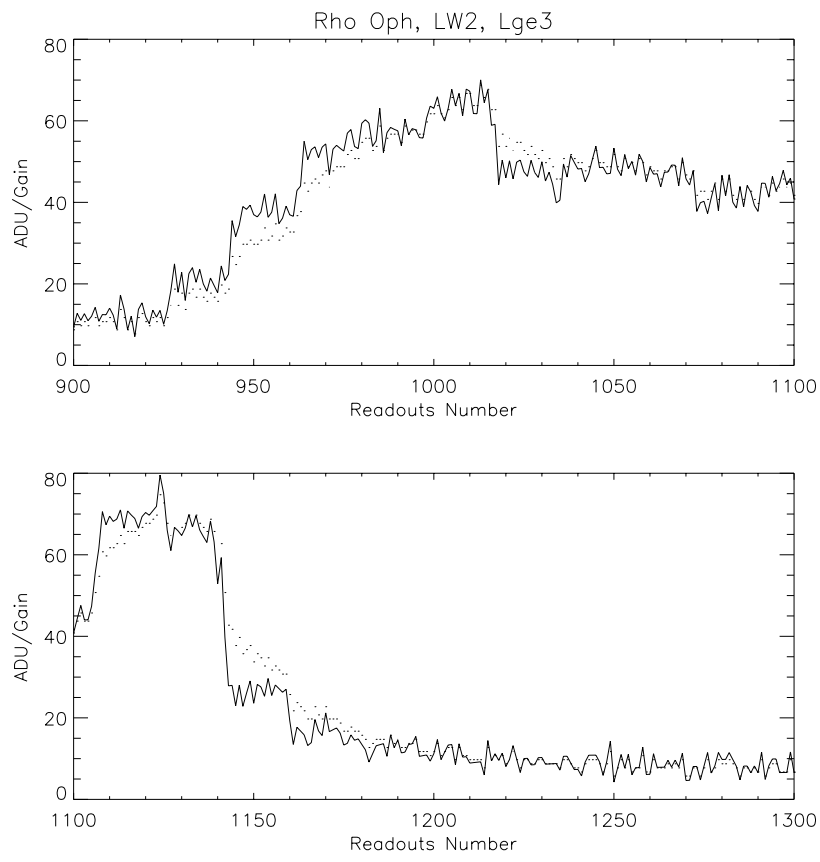

Fig. 6. Example of transient correction on diffuse emission, taken from the observations of $\rho$ Oph (Abergel et al. 1996) made with the LW2 filter and with a 3 arcsec pixel field of view lens. Dots: non corrected observation. Solid line: transient corrected observation. Upper panel: a typical time series when CAM points from zodiacal background to a diffuse emission region. Lower panel: a typical time series when CAM points from a diffuse emission region to the zodiacal background, leading to a decreasing transient

resulting correction is not perfect, since it is limited in precision by the precision of the model of the CAM-LW response we use which is obviously oversimplified. In particular, for large flux steps, the memory effect is predicted to be damped too fast. The model of the response we use is approximate. We have already pointed out that upward and downward transients resulting from large flux steps (typically $80 \mathrm{ADU} \mathrm{g}^{-1} \mathrm{~s}^{-1}$ ) are not symmetrical and depart from a simple exponential behavior, while the model is symmetrical and exponential. The photometric accuracy of the LW channel of ISOCAM is actually of $5-10 \%$ after applying the transient correction algorithm we have presented in this paper. It is not better for strong sources than for faint sources, because of the over-simplification of the model of the response we use. We are currently working on algorithms to correct ISOCAM-LW data from transient effects taking into account the charge coupling, which introduces most of the features in the response curves not taken into account at the present time.

\subsection{Examples}

Figure 6 shows an example of transient correction in the two general cases of observation of diffuse emission: when 


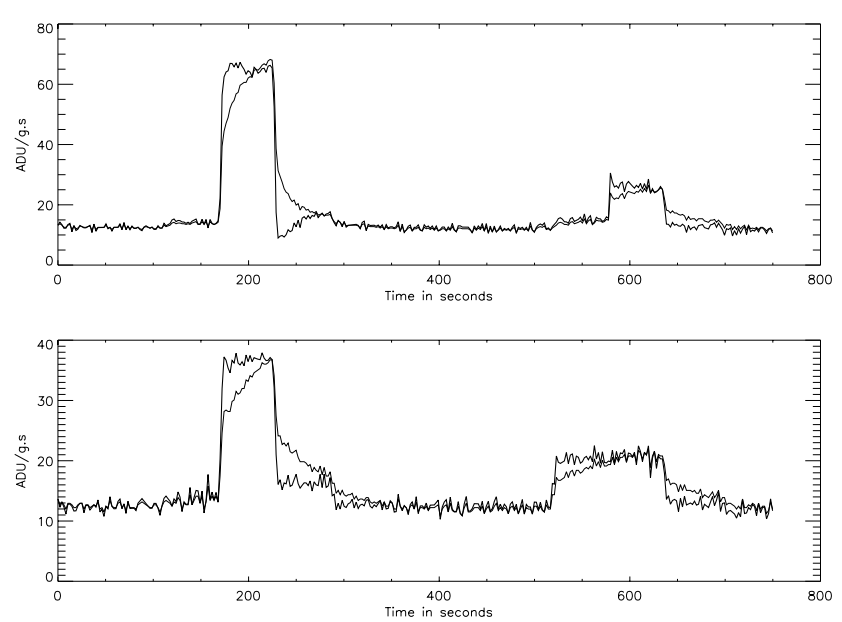

Fig. 7. Pixel response in ADU Gain ${ }^{-1} \mathrm{~s}^{-1}$ versus time during an observation on Centaurus A using the LW3 filter and the 3 arcsec PFOV lens. In both cases the thin line is the original data dark-corrected and deglitched, while the thick line is the transient corrected data. As mentioned in the text, data previous to the first frame of the cube are assumed to have a constant value, identical to that found in the first frame. Hence there is little difference between the original and corrected data at the start of the observation. The upper panel shows how the formula used to described the transient behavior of the detector fails on strong steps: it creates an overshoot, or undershoot, for respectively upward steps, or downward steps. In the lower panel, where the flux steps are smaller (the $y$ axis scale is half that of the upper panel) the overshoots and undershoots are gone and the correction is much cleaner

CAM first points at a region of zodiacal background, then to a diffuse emission region, then back. The correction appears to be good. Various levels of flux that were blurred due to transients in the original data, because CAM did not spend enough time on each position to become stabilized, become obvious on the corrected data, even for small flux steps. As mentioned earlier, the representation that has been adopted to describe the response of ISOCAMLW is not fully adequate. In particular it is unable to correctly predict the behavior of transients due to strong flux steps. This is illustrated in Fig. 7 where we show data coming from two pixels that see the brightest region of an observation of Centaurus A in the LW3 filter and with the $3^{\prime \prime}$ pixel field-of-view lens. In the upper panel a strong step occurs and as a result, the corrected data (thick line) show an overshoot for the upward transient and an undershoot for the downward transient. This effect is much reduced in the lower panel where the maximum flux steps are $1 / 2$ those in the upper panel.

\subsection{A short word on fitting methods}

A number of fitting algorithms have been created to tackle the problem of transient correction (see, e.g., van Buren
1996; Lari 1997). It is not our point to elaborate on the merit of these methods, yet we can use the modeling of ISOCAM's response developed above to investigate the possibility of fitting the observed signal rather than inverting it. A fit offers the further possibility of letting some of the model parameters vary.

We therefore selected a number of observations that satisfy the following constraint: the source is extended but does not fill the array, and it is observed using a long raster. Thus it is easy to find pixels that will spend enough time on the background so that they are nearly stabilized, see part of the source for one raster position, and then observe the background again. Therefore the incoming flux history is very simple and can be written as: $l_{\text {back }} \rightarrow l_{\text {src }} \rightarrow l_{\text {back }}$, where $l_{\text {back }}$ is the background flux, and $l_{\text {src }}$ the source flux. Given the selection criteria we have applied, $l_{\text {back }}$ can safely be assumed to be the flux measured on the pixel prior to the raster position that sees the source. The instant at which the fluxes change are also easily derived from the data given the existence of the fast component in the pixel's response.

We have then tried to fit the response model (19) to the observed signal, letting the model parameters $r$ and $\alpha$ vary. We thus have three parameters to derive from the fit: $l_{\text {src }}, r$ and $\alpha$. The results of this study have been presented elsewhere (Sauvage 1997) but we summarize them here.

1. Very satisfactory fits can be obtained for a range of $l_{\mathrm{src}}$ going from a few to $\simeq 60 \mathrm{ADU} \mathrm{g}^{-1} \mathrm{~s}^{-1}$. Above that level we observe that the transient starts to significantly depart from the predicted behavior.

2. No single couple of values for $r$ and $\alpha$ can fit all the transients we selected. That is still true when we restrict the study to one pixel, in order to eliminate pixel to pixel variations.

3. $\alpha$ was found to depend on $l_{\text {src }}$ in a way that is contrary to the expectations, i.e. the smaller the flux the faster the stabilization.

Point (3) is in total contradiction with the transient behavior deduced from ground-based controlled experiment; in these we observed that the lower the flux, the longer the detector took to stabilize. It therefore casts serious doubts on the values of $l_{\mathrm{src}}$ found in the fit.

The main conclusion of this study is thus that the quality of the fit cannot guarantee that the result is photometrically correct. We thus caution observers against fitting methods for transient correction as they offer little control on the validity of the resulting photometry.

In the present case, we interpret the discrepancy between point (3) and our experimental knowledge of the detector as beeing due to an oversimplification of the response function (Eq. 19): it does not account for a slow part in the transient behavior and fails to reproduce transients observed on strong sources (see Fig. 7). Nevertheless, the values of $r$ and $\alpha$ are such that (1) memory effects are quite significantly reduced, and (2) residual 
photometric errors are limited and can be quantified to $\simeq 10 \%$.

\section{Jitter}

Jitter correction can be done by optimizing the correlation function between two successive frames. Jitter standard deviation is around 0.1 arcsec. This effect is rather weak, yet, as mentioned in Sect. 2, it can alter the flux due to incorrect deglitching. Apart from this effect, and for most observations, jitter correction is not needed.

\section{Field of view distortion correction}

Field distortion in ISOCAM is mostly due to the off-axis mirror that directs the light beam toward each detector and to the fact that the ISOCAM field of view is an offaxis part of the full FOV of the ISO telescope. The field distortion was measured for the LW channel $6^{\prime \prime}$ and $3^{\prime \prime}$ lenses, using calibration observations of fields that contained many stars. No measurements have been made for the $1.5^{\prime \prime}$ lens because they are difficult to perform as the amplitude of the satellite jitter is of the order of the quantities to be measured. Since the distortion with the $1.5^{\prime \prime}$ lens is predicted to be negligible, no error would be made if it is not taken into account.

ISOCAM also suffers from lens wheel jitter. In order to avoid any mechanical blocking, the gear wheel has been designed with a small play. Therefore, the position at which the lens stops is not fixed.

It has been shown by the CAM Instrument Dedicated Team that there are only two broad families of positions that the lens wheel can take for a commanded position, and it is suspected that the wheel stops at either side of the play. This can be very easily detected by close inspection of the flat field derived from the data: the leftmost column of the detector receives very little light. This is called the "left" position. This jitter results in an offset of about 1.2 pixels of the optical axis, thus $\approx 7^{\prime \prime}$ with the $6^{\prime \prime}$ lens. It also modifies the distortion pattern and therefore the latter has been measured for both positions. The measurement method is discussed in Aussel (1997).

Following the work done on the HST WFPC by Holtzman et al. (1995), each measurement is fitted with a general polynomial of degree 3 , that is:

$$
\begin{aligned}
x_{\mathrm{c}}= & a_{0}+a_{1} x+a_{2} y+a_{3} x^{2}+a_{4} x y+a_{5} y^{2} \\
& +a_{6} x^{3}+a_{7} x^{2} y+a_{8} x y^{2}+a_{9} y^{3} \\
y_{\mathrm{c}}= & b_{0}+b_{1} x+b_{2} y+b_{3} x^{2}+b_{4} x y+b_{5} y^{2} \\
& +b_{6} x^{3}+b_{7} x^{2} y+b_{8} x y^{2}+b_{9} y^{3}
\end{aligned}
$$

where $x_{\mathrm{c}}$ and $y_{\mathrm{c}}$ are the positions on the ISOCAM LW array in pixels, corrected for distortion, while $x$ and $y$ are the non-corrected ones. Figure 8 shows a map of the distortion of the LW channel of ISOCAM with the $6^{\prime \prime}$ lens,

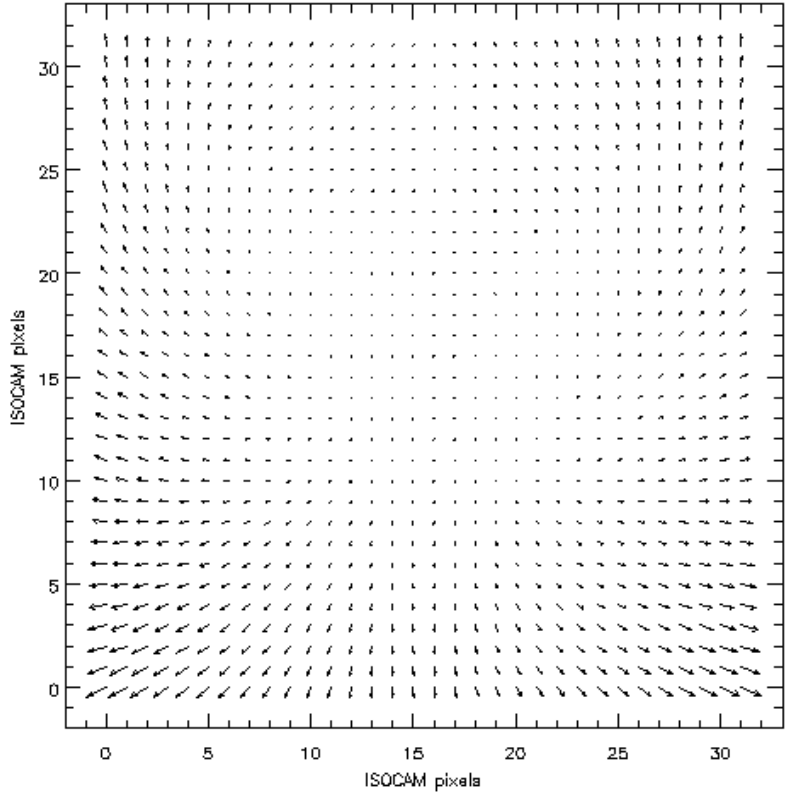

Fig. 8. ISOCAM field of view distortion (6 arcsec lens)

where each vector starts from where the center of a pixel should fall were there no distortion and ends at its actual position. The length of the vectors are at the scale of the plot. At the lower corners of the array (lines 0 to 5 ), the effect is greater than one pixel.

Since the pixel size is not uniform on the sky, the pixels at the edges of the array cover a wider surface. Therefore, a new flat-field correction has to be applied in order to account for it. This flat-field is of the form:

$F_{i, j}=\frac{S_{16,16}}{S_{i, j}}$

with $S_{i, j}$, the surface on the sky of pixel $i, j$. The pixel $(16,16)$, being the center pixel of ISOCAM LW array and therefore the less distorted, has been taken as reference.

This is where distortion corrections stop in the case of staring observations. In a raster mode, where an image has to be constructed from the coaddition of many smaller ones, the processing continues as follows: each raster sub-image is projected on the raster map, using a flux-conservative shift and add algorithm. The intersecting surface $S_{(x, y, i, j)}$ of each sky pixel of the raster map with each ISOCAM pixel is computed. The flux in the pixel $(x, y)$ of the raster map is therefore:

$R_{x, y}=\frac{\sum_{\text {pointings }} S_{(x, y, i, j)} \sqrt{N_{i, j}} I_{i, j}}{\sum_{\text {pointings }} S_{(x, y, i, j)} \sqrt{N_{i, j}}}$.

Assuming Gaussian noise distributions for pixels, the noise map is:

$\sigma_{x, y}=\sqrt{\frac{\sum_{\text {pointings }} S_{(x, y, i, j)}^{2} N_{i, j} \sigma_{i, j}^{2}}{\sum_{\text {pointings }} S_{(x, y, i, j)}^{2} N_{i, j}}}$.

Where $R_{x, y}$ is the value of the final raster map at $(x, y)$, $S_{(x, y, i, j)}$ is the intercepted surface between ISOCAM pixel 

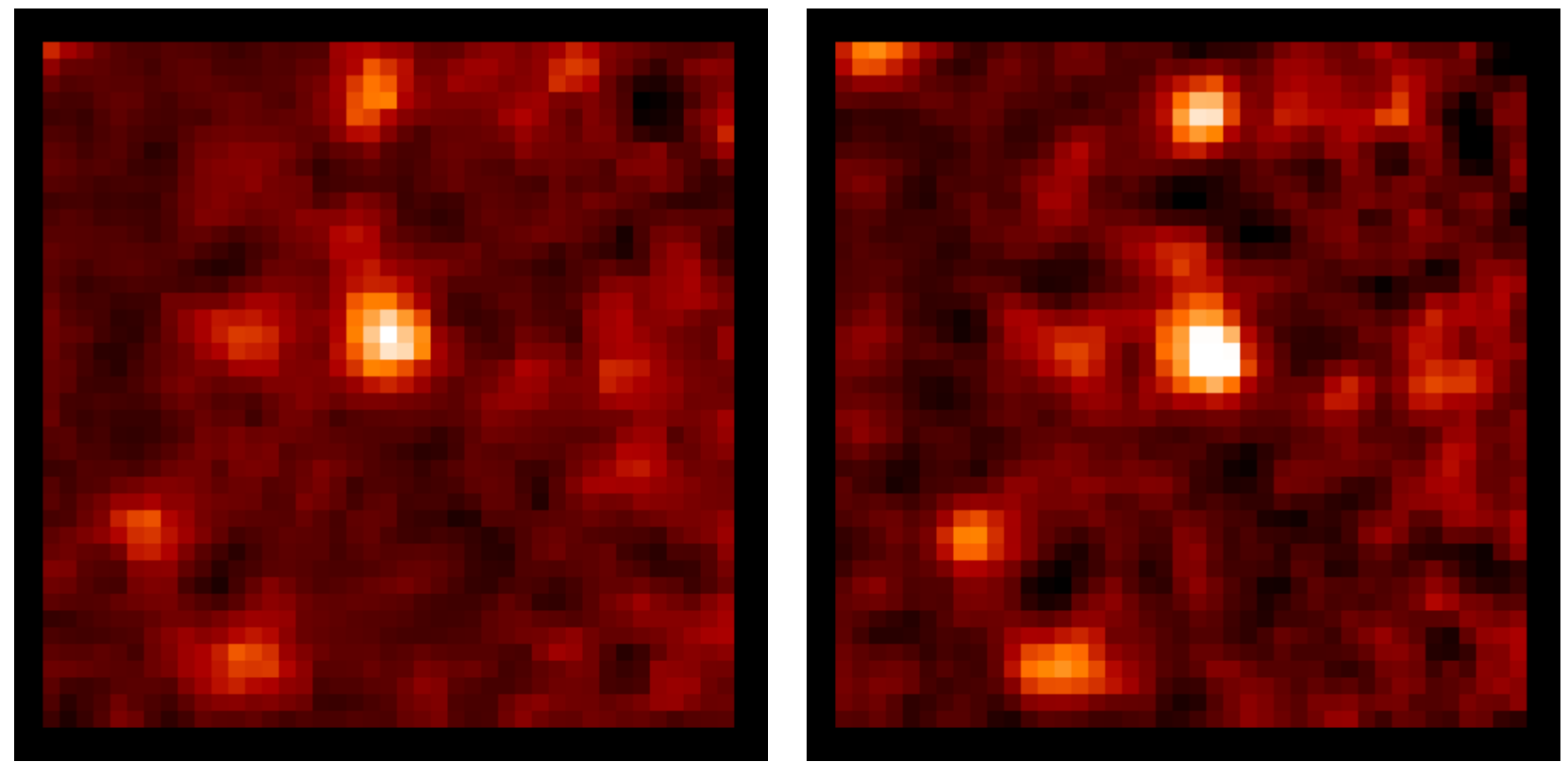

Fig. 9. Zoom on a part of the ISOCAM Hubble Deep Field observation at $15 \mu \mathrm{m}$. The same processing was applied to the two images, except for the correction of the distortion. Cuts on images are at the same level. Pixel size is $2^{\prime \prime}$

$(i, j)$ and raster map pixel $(x, y)$ and $N_{i, j}$ is the number of readouts averaged together to produce $I_{i, j}$, the image of the raster pointing. The computation of $S_{(x, y, i, j)}$ is derived from the algorithm used by Fruchter et al. in their "drizzle" IRAF task. The files containing the coefficients for the distortion correction are available from the authors under a format accepted by this task.

Figure 9 shows a zoom on a part of the ISOCAM Hubble Deep Field observation at $15 \mu \mathrm{m}$. The same processing was applied to the two images, except for the correction of the distortion (pixel size is $2^{\prime \prime}$ ).

\section{Conclusion}

We have presented in this paper a way to treat some ISOCAM data. A number of other algorithms have been developed and tested, but we have presented here only those which produce the best results. Other independent reduction techniques for faint source detection with ISOCAM are detailed in Aussel et al. (1997), Désert et al. (1997), and Starck et al. (1997). Imperfect transient correction effects actually dominates the photometric uncertainty of the LW channel of ISOCAM (about $5-10 \%$ ).

The methods described above are available using the Cam Interactive Analysis (Ott et al. 1996) at the ISOCAM Data Analysis Center at CEA Saclay, or at Vilspa.

Acknowledgements. We wish to thank Suzanne Madden for useful discussions and comments.

\section{References}

Abergel A., et al., 1998 (in preparation)

Abergel A., et al., 1996, "Isocam mapping of the $\rho$ Ophiuchi main cloud", A\&A letters

Aussel H., 1997, "ISOCAM LW channel field of view distortion",

http:///isowww.estec.esa.nl:80/instr/CAM/cal_wksp/

Aussel H., Elbaz D., Starck J.L., Cesarsky C., 1997, Moriond Astrophysics Meeting on Extragalactic Astronomy in the Infrared, Les Arcs, France

Biviano A., Sauvage M., Roman P., Boulade O., Gallais P., Okumura K., 1998, "The ISOCAM dark current calibration report", ESA report (in preparation)

Cesarsky C.J., et al., 1996, "ISOCAM in flight", å315L32

Désert F.-X., Puget J.-L., Clements D.L., Pérault M., Abergel A., Bernard J.-P., Cesarsky C., 1998, A\&A (submitted)

Fouks B.I., Schubert J., 1995, "Precise theoretical description of the photoresponse for detectors of ISOPHOT's Si:Ga array", Proc. SPIE 2475487

Haegel N.M., Newton C., Simoes J.C., White A.M., 1996, "Modeling of the transient response in far infrared photoconductors", Proc. 30th ESLAB Symp. Submillimeter and Far-Infrared Space Instrumentation, ESA SP-388, 15-20

Holtzman J.A., Burrows C.J., Casertano S., Hester J.J., Trauger J.T., Watson A.M., Worthey G., 1995, PASP 107, 156

Lari C., 1997, "report on CAM transients", http:///isowww.estec.esa.nl:80/instr/CAM/cal_wksp/

Ott S., et al., 1996, ASP Conf. Syst. 125, 1997

Sauvage M., 1997, "Properties of ISOCAM transients", http:///isowww.estec.esa.nl:80/instr/CAM/cal_wksp/

Schubert J., Fouks B.I., Lemke D., Wolf J., 1995, "Transient Response of ISOPHOT Si:Ga Infrared Photodetectors: Experimental results and Application of the Theory of nonstationary processes", Proc. SPIE 2553461 
Starck J.L., Murtagh F., Bijaoui A., 1998, "Image Processing and Data Analysis: the Multiscale Approach". Cambridge University Press, Cambridge (UK)

Starck J.L., Aussel H., Elbaz D., Cesarsky C., 1997, Moriond Astrophysics Meeting on Extragalactic Astronomy in the Infrared, Les Arcs, France

Starck J.L., Claret A., Siebenmorgen R., 1996, "Isocam Data Calibration", CEA Saclay Technical Report

Starck J.L., Murtagh F., 1998, "Automatic Noise Estimation from the Multiresoluiton Support", PASP 110, pp. 193-199 Starck J.L., Pantin E., 1996, "Second Order Dark Correction", CEA Saclay Technical Report

Starck J.L., Murtagh F., Pirenne B., Albrecht M., 1996, PASP 108, pp. 446-455

Van Buren D., Aussel H., 1996, "ISOCAM-LW dark frames are simply related", IPAC Technical Report

Van Buren D., 1996, "Global modeling of ISOCAM-LW", IPAC technical report 